\title{
Vitamin D and Multiple Sclerosis: Correlation, Causality, and Controversy
}

\author{
Joost Smolders ${ }^{1,2}$ \\ ${ }^{1}$ Division of Clinical and Experimental Immunology, Department of Internal Medicine and School for Mental Health and Neuroscience, \\ Maastricht University Medical Center, Universiteitssingel 50, P.O. Box 616, 6200 MD Maastricht, The Netherlands \\ ${ }^{2}$ Academic MS Center Limburg, Orbis Medical Center, P.O. Box 5500, 6130 MB Sittard, The Netherlands
}

Correspondence should be addressed to Joost Smolders, j.smolders@mumc.nl

Received 13 September 2010; Accepted 14 September 2010

Academic Editor: Sreeram Ramagopalan

Copyright (c) 2011 Joost Smolders. This is an open access article distributed under the Creative Commons Attribution License, which permits unrestricted use, distribution, and reproduction in any medium, provided the original work is properly cited.

\begin{abstract}
The last years, many studies reported associations between correlates of vitamin D exposure and several correlates of multiple sclerosis (MS) disease activity. This review discusses studies on vitamin D status, Expanded Disability Status Scale (EDSS) score, and relapse activity of MS. Furthermore, several considerations for intervention studies on vitamin D supplementation in MS are provided.
\end{abstract}

Vitamin D is hot in multiple sclerosis (MS) research. The geographical distribution of MS prevalence, which increases when approaching the poles, sparked the interest in a poor vitamin D exposure as a risk factor for developing MS [1]. Now, half a century of research further, a large body of clinical observations and experimental work in vitro and in animal models of MS has been reported [2]. As reviewed recently, a limited exposure to sunlight and other correlates of vitamin D exposure has been associated consistently with an increased risk on developing MS [3]. The prospect of a potential tool to prevent MS is tempting, yet challenging to investigate in an intervention study since it would require a huge population to measure any effect on MS incidence. However, vitamin D supplementation has not only been proposed to prevent MS, but also to attenuate disease activity of MS [4].

In patients with established MS, earliest observations show a seasonal fluctuation of several disease characteristics. In Switzerland, more relapses were recorded during winter and spring than during summer [5]. More gadolinium enhancing lesions on T1 MRI have been reported in Germany in spring and early summer, and less in autumn [6]. Regrettably, these observations were not related directly to serum concentrations of 25-hydroxyvitamin D (25(OH)D), the vitamin $\mathrm{D}$ metabolite that reflects vitamin $\mathrm{D}$ status best
[7], and prove difficult to reproduce. Other studies did correlate measures of MS severity directly with vitamin D status. The most widely used score to quantify disability due to MS is the Expanded Disability Status Scale (EDSS) [8]. EDSSscore has been reported to correlate negatively with serum 25(OH)D levels $[9,10]$. Van der Mei et al. showed elegantly that EDSS-score also correlated negatively with recent sun exposure [10]. Therefore, it remains to be seen whether vitamin D status contributes to disability progression in MS, since (prospective) studies on this issue are at present lacking. Interestingly, however, Burton et al. performed a small phase I/II study which compared two groups of MS patients on supplementation with either high (up to $40.000 \mathrm{IU} / \mathrm{d}$ ) or low doses $(\leq 4.000 \mathrm{IU} / \mathrm{d})$ of vitamin $\mathrm{D}_{3}$ for 52 weeks [11]. They reported a smaller proportion of patients with any progression on the EDSS-scale in the high- versus lowdose vitamin D group (2/25 versus 9/24, resp.). Although premature, this finding encourages further research on the effect of vitamin D on disease progression of MS. Relapse activity has also been related to vitamin D status. During relapse, lower serum $25(\mathrm{OH}) \mathrm{D}$ levels have been reported in several MS populations, when compared to remission of MS [12-14]. However, also this information is prone to many interpretations. We observed that patients with $<5$ years relapsing remitting $\mathrm{MS}$ (RRMS) and $\geq 1$ relapses in the 2 
years prior to serum sampling had markedly lower serum $25(\mathrm{OH}) \mathrm{D}$ levels when compared to relapse-free patients [9]. Interestingly, a large prospective longitudinal study in RRMS patients with a wider range of disease duration showed that an increase of serum 25(OH)D levels with $10 \mathrm{nmol} / \mathrm{L}$ was associated with a $9-12 \%$ reduction of the hazard on relapses [15]. Additionally, a prospective study in children with RRMS calculated a comparable $14 \%$ decrease in hazard on relapses for each $10 \mathrm{nmol} / \mathrm{L}$ increase of vitamin D status [16]. These studies suggest that supplementation with vitamin D may protect RRMS patients against relapses. Interestingly, although not statistically significant, a smaller proportion of patients with relapses was described by Burton et al. in the high- versus low-dose vitamin $\mathrm{D}_{3}$ supplementation group [11].

Altogether, the evidence that is available on vitamin D status and disease activity of MS shows a consistent picture. To consolidate a possible role of vitamin D supplementation in the treatment of MS, randomized placebocontrolled clinical trials are needed. Supplementation of high doses of vitamin D could easily elevate serum $25(\mathrm{OH}) \mathrm{D}$ levels with $100 \mathrm{nmol} / \mathrm{L}[11,17]$. When a linear relationship between vitamin $\mathrm{D}$ status and reduction of relapse-risk is assumed [15], the effect of this intervention on relapse-risk could be substantial $[15,16]$. However, there are several considerations. First of all, vitamin D status predicts the hazard on relapses, but vitamin D status itself is dependent on sun exposure and outdoor physical activity. There is some evidence available that both these factors contribute individually to disease activity of MS [18]. Additionally, the inverse linearity of the relationship between vitamin D status and hazard for relapses in the supraphysiological range of serum 25(OH)D levels is uncertain [15]. Therefore, the effect in intervention studies may not be as large as predicted from observational studies. Secondly, the amount of vitamin $\mathrm{D}$ which should be supplemented is uncertain. In most clinical trials with a new treatment, the difference between presence and absence of a specific substance is compared. In studies on vitamin $\mathrm{D}$, an effect of supraphysiological versus physiological levels of vitamin $\mathrm{D}$ exposure will be assessed. Supplementation of low doses may fail to reduce the risk on relapses significantly when compared to the physiological range of vitamin D status in control groups. This problem could be tackled by selective inclusion of patients with a poor vitamin D status. Alternatively, high doses of vitamin D could be supplemented. Although shortterm exposure to high doses of vitamin D showed no sideeffects $[11,17]$, there are some concerns on long-term safety and efficacy. Thirdly, the groups of patients which should be supplemented with vitamin $\mathrm{D}$ are ill defined. The most fundamental choice is the inclusion of either RRMS or progressive MS patients. Progressive MS patients display the poorest vitamin D status [9], yet an effect of vitamin D on EDSS progression is less well consolidated. Since an effect on relapse activity is most likely $[15,16]$, studies in RRMS patients are most obvious. Mechanistic approaches may also help to specify the patients to be included. We showed that, in RRMS patients with a short disease duration of $<5$ years, vitamin D status correlated with peripheral
T cell homeostasis [19]. Additionally, supplementation of vitamin $\mathrm{D}$ reduced the $\mathrm{T}$ cell proliferative response to myelin antigens in RRMS patients [11]. Alternatively, $25(\mathrm{OH}) \mathrm{D}$ is also present in the central nervous system of MS patients and may be involved in local immune regulatory systems in progressive MS [20]. Fourthly, response of patients to vitamin $\mathrm{D}$ supplementation may depend upon the genetic profile of both vitamin D-related genes [21] and MHC class II genotype [22]. Intervention studies should allow analyses on genetic profiles associated with response to vitamin D. Lastly, studies should not only focus on classical outcomes as MRI parameters, relapse activity, and disability progression. Other symptoms found in MS have also been associated with a poor vitamin $\mathrm{D}$ status, including presence of depressive symptoms [23] and cognitive decline [24]. Including also these parameters could provide a complete overview of the impact of vitamin D supplementation in MS.

There are many different opinions about vitamin $\mathrm{D}$ in MS, ranging from scepticism to a firm belief in the new panacea. These opinions reflect the current state of evidence: there are a lot of indications for a therapeutic role of vitamin D in MS, but these remain all circumstantial evidence. Everyone will agree, however, that these indications provide at least an excellent reason to develop well-designed clinical trials on vitamin D supplementation in MS. Currently first clinical trials are starting up and will undoubtedly cast more light on the issue.

\section{Acknowledgments}

The author received consultancy fee from and serves in a steering committee for Merck Serono. He received fee for travelling from Biogen Idec. The author thanks prof. Dr. R. Hupperts and Dr. J. Damoiseaux for their comments on the manuscript.

\section{References}

[1] E. D. Acheson, C. A. Bachrach, and F. M. Wright, "Some comments on the relationship of the distribution of multiple sclerosis to latitude, solar radiation, and other variables," Acta Psychiatrica Scandinavica, vol. 35, no. 147, pp. 132-147, 1960.

[2] J. Smolders, J. Damoiseaux, P. Menheere, and R. Hupperts, "Vitamin D as an immune modulator in multiple sclerosis, a review," Journal of Neuroimmunology, vol. 194, no. 1-2, pp. 7-17, 2008.

[3] A. Ascherio, K. L. Munger, and K. C. Simon, "Vitamin D and multiple sclerosis," Lancet Neurology, vol. 9, no. 6, pp. 599-612, 2010.

[4] P. Goldberg, M. C. Fleming, and E. H. Picard, "Multiple sclerosis: decreased relapse rate through dietary supplementation with calcium, magnesium and vitamin D," Medical Hypotheses, vol. 21, no. 2, pp. 193-200, 1986.

[5] R. Wüthrich and H. P. Rieder, "The seasonal incidence of multiple sclerosis in Switzerland," European Neurology, vol. 3, no. 5, pp. 257-264, 1970.

[6] D. P. Auer, E. M. Schumann, T. Kümpfel, C. Gössl, and C. Trenkwalder, "Seasonal fluctuations of gadolinium-enhancing magnetic resonance imaging lesions in multiple sclerosis," Annals of Neurology, vol. 47, no. 2, pp. 276-277, 2000. 
[7] B. W. Hollis, "Assessment of vitamin D nutritional and hormonal status: what to measure and how to do it," Calcified Tissue International, vol. 58, no. 1, pp. 4-5, 1996.

[8] J. F. Kurtzke, "Rating neurologic impairment in multiple sclerosis: an expanded disability status scale (EDSS)," Neurology, vol. 33, no. 11, pp. 1444-1452, 1983.

[9] J. Smolders, P. Menheere, A. Kessels, J. Damoiseaux, and R. Hupperts, "Association of vitamin D metabolite levels with relapse rate and disability in multiple sclerosis," Multiple Sclerosis, vol. 14, no. 9, pp. 1220-1224, 2008.

[10] I. A. F. van der Mei, A.-L. Ponsonby, T. Dwyer et al., "Vitamin D levels in people with multiple sclerosis and community controls in Tasmania, Australia," Journal of Neurology, vol. 254, no. 5, pp. 581-590, 2007.

[11] J. M. Burton, S. Kimball, R. Vieth et al., "A phase I/II dose-escalation trial of vitamin D3 and calcium in multiple sclerosis," Neurology, vol. 74, no. 23, pp. 1852-1859, 2010.

[12] J. Correale, M. C. Ysrraelit, and M. I. Gaitán, "Immunomodulatory effects of Vitamin D in multiple sclerosis," Brain, vol. 132, no. 5, pp. 1146-1160, 2009.

[13] M. Soilu-Hänninen, L. Airas, I. Mononen, A. Heikkilä, M. Viljanen, and A. Hänninen, "25-hydroxyvitamin D levels in serum at the onset of multiple sclerosis," Multiple Sclerosis, vol. 11, no. 3, pp. 266-271, 2005.

[14] M. Soilu-Hänninen, M. Laaksonen, I. Laitinen, J.-P. Erälinna, E.-M. Lilius, and I. Mononen, "A longitudinal study of serum 25-hydroxyvitamin D and intact parathyroid hormone levels indicate the importance of vitamin D and calcium homeostasis regulation in multiple sclerosis," Journal of Neurology, Neurosurgery and Psychiatry, vol. 79, no. 2, pp. 152-157, 2008.

[15] S. Simpson Jr., B. Taylor, L. Blizzard et al., "Higher 25hydroxyvitamin $\mathrm{D}$ is associated with lower relapse risk in multiple sclerosis," Annals of Neurology, vol. 68, no. 2, pp. 193 203, 2010.

[16] E. M. Mowry, L. B. Krupp, M. Milazzo et al., "Vitamin D status is associated with relapse rate in pediatric-onset multiple sclerosis," Annals of Neurology, vol. 67, no. 5, pp. 618-624, 2010.

[17] S. M. Kimball, M. R. Ursell, P. O’Connor, and R. Vieth, "Safety of vitamin $\mathrm{D}_{3}$ in adults with multiple sclerosis," American Journal of Clinical Nutrition, vol. 86, no. 3, pp. 645-651, 2007.

[18] B. R. Becklund, K. S. Severson, S. V. Vang, and H. F. DeLuca, "UV radiation suppresses experimental autoimmune encephalomyelitis independent of vitamin D production," Proceedings of the National Academy of Sciences of the United States of America, vol. 107, no. 14, pp. 6418-6423, 2010.

[19] J. Smolders, M. Thewissen, E. Peelen et al., "Vitamin D status is positively correlated with regulatory $\mathrm{T}$ cell function in patients with multiple sclerosis," PLOS ONE, vol. 4, no. 8, article e6635, 2009.

[20] T. Holmøy, S. M. Moen, T. A. Gundersen et al., “25hydroxyvitamin $\mathrm{D}$ in cerebrospinal fluid during relapse and remission of multiple sclerosis," Multiple Sclerosis, vol. 15, no. 11, pp. 1280-1285, 2009.

[21] S.-M. Orton, A. P. Morris, B. M. Herrera et al., "Evidence for genetic regulation of vitamin D status in twins with multiple sclerosis," American Journal of Clinical Nutrition, vol. 88, no. 2, pp. 441-447, 2008.

[22] S. V. Ramagopalan, N. J. Maugeri, L. Handunnetthi et al., "Expression of the multiple sclerosis-associated MHC class II allele HLA-DRB1*1501 is regulated by vitamin D," PLoS Genetics, vol. 5, no. 2, Article ID e1000369, 2009.

[23] S. Knippenberg, Y. Bol, J. Damoiseaux, R. Hupperts, and J. Smolders, "Vitamin D status in MS patients is negatively correlated with depression, but not with fatigue," Acta Neurologica Scandinavica. In press.

[24] D. J. Llewellyn, I. A. Lang, K. M. Langa et al., "Vitamin D and risk of cognitive decline in elderly persons," Archives of Internal Medicine, vol. 170, no. 13, pp. 1135-1141, 2010. 


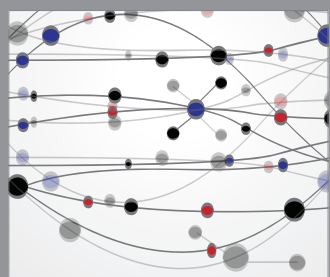

The Scientific World Journal
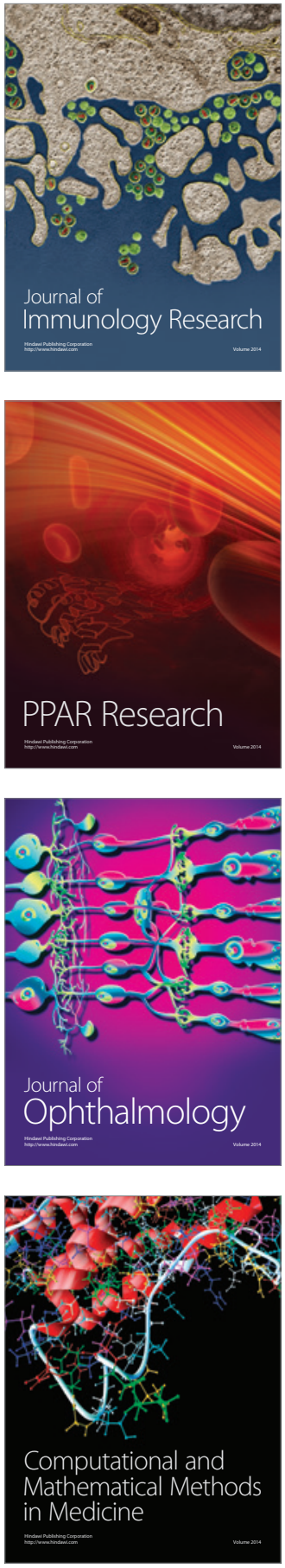

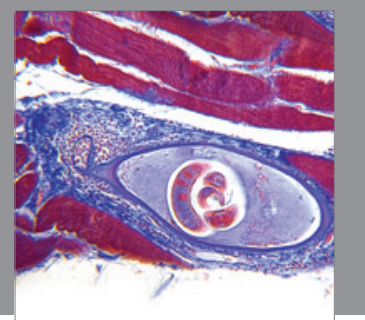

Gastroenterology

Research and Practice
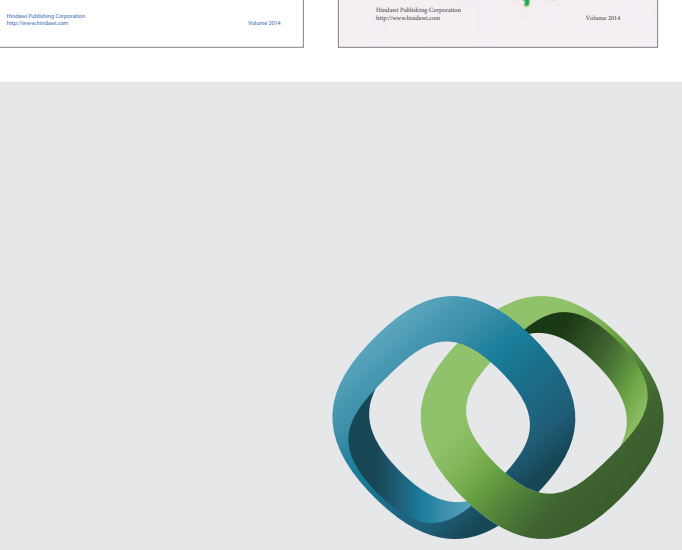

\section{Hindawi}

Submit your manuscripts at

http://www.hindawi.com
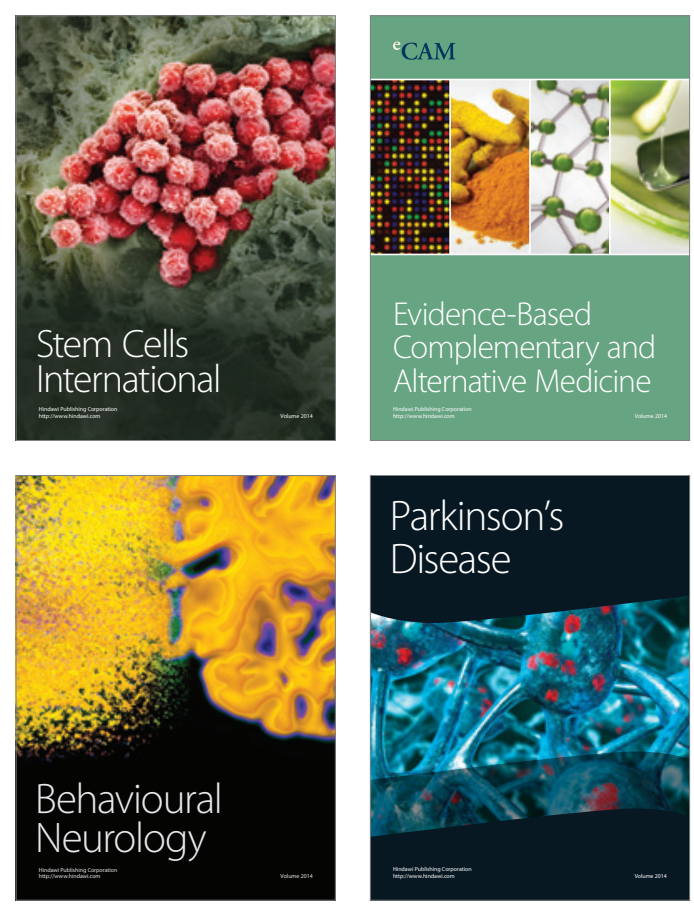

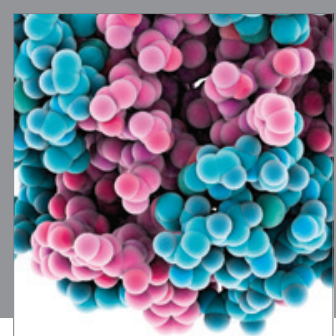

Journal of
Diabetes Research

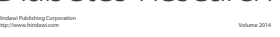

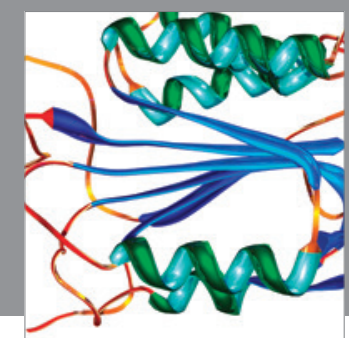

Disease Markers
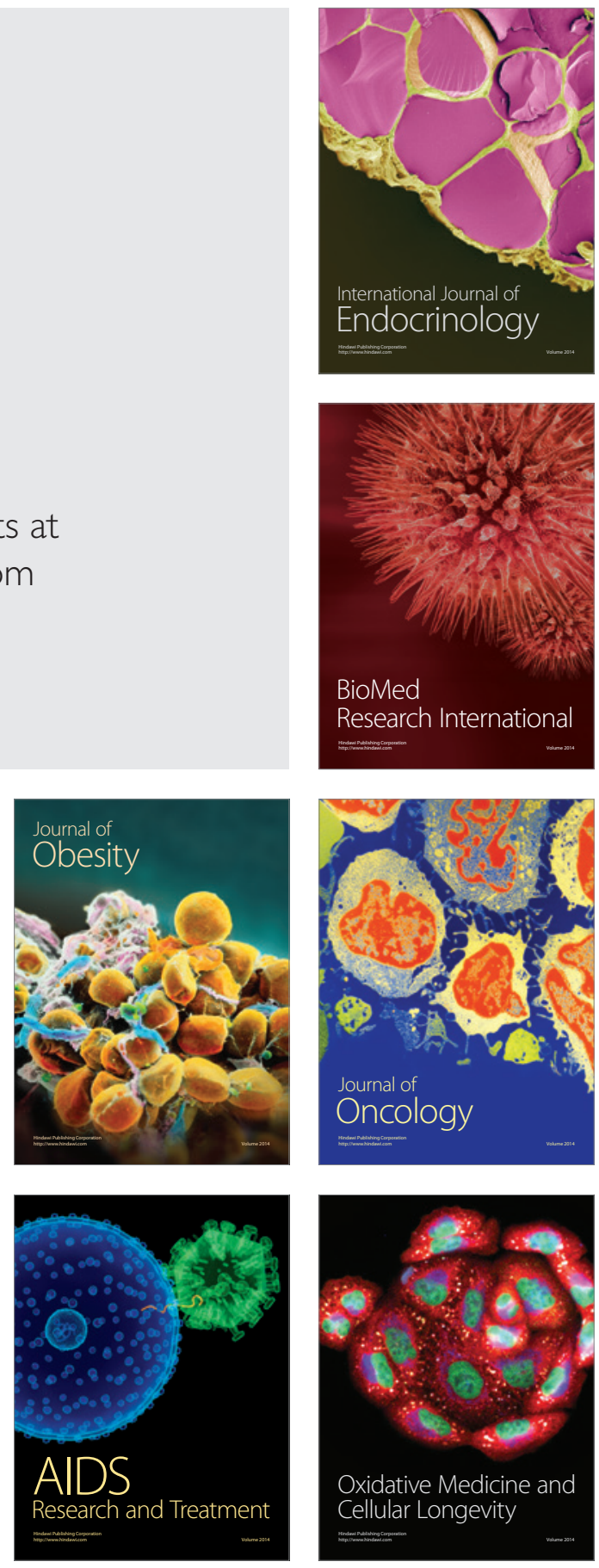